\title{
TITLE:
}

\section{Break conductance of noble metal contacts}

$\operatorname{AUTHOR}(S):$

Fujii, A; Tsutsui, M; Kurokawa, S; Sakai, A

CITATION:

Fujii, A ... [et al]. Break conductance of noble metal contacts. PHYSICAL REVIEW B 2005, 72(4): 045407.

ISSUE DATE:

2005-07

URL:

http://hdl.handle.net/2433/39874

RIGHT:

Copyright 2005 American Physical Society 
PHYSICAL REVIEW B 72, 045407 (2005)

\title{
Break conductance of noble metal contacts
}

\author{
Akihiro Fujii, Makusu Tsutsui, Shu Kurokawa, and Akira Sakai* \\ International Innovation Center, Kyoto University, Sakyo-ku, Kyoto 606-8501 Japan \\ (Received 22 October 2004; revised manuscript received 22 February 2005; published 6 July 2005)
}

\begin{abstract}
We have studied the instability of noble metal contacts under high biases (currents) by measuring their break conductance, a conductance just prior to the contact break. At high contact currents $(\gtrsim 1 \mathrm{~mA})$, the break conductance distribution shows a broad single peak located at $\sim(10-50) G_{0}\left(G_{0} \equiv 2 e^{2} / h\right.$ is the quantum unit of conductance). From the observed peak shift with the contact current, we showed that the contact break of noble metals most likely takes place when the contact current density reaches a critical value $(6-9) \times 10^{10} \mathrm{~A} / \mathrm{cm}^{2}$. Noble metal contacts thus make a current-induced break under high currents. At lower biases (currents), a contact tends to break off at one of its preferred contact geometries. We found that the $1 G_{0}$ contact is always strongly preferred at the contact break of $\mathrm{Au}$, while contacts of $1 G_{0}, 2.5 G_{0}, 5 G_{0}$, and $7.5 G_{0}$ appear at the break of $\mathrm{Ag}$ and $\mathrm{Cu}$ contacts. Preference of these contact geometries of $\mathrm{Ag}$ and $\mathrm{Cu}$ at the contact break is weakly affected by the contact bias (current).
\end{abstract}

DOI: 10.1103/PhysRevB.72.045407

PACS number(s): 73.63.Rt, 81.07.Lk, 66.30.Qa, 73.40.Jn

\section{INTRODUCTION}

In recent years, a number of theoretical and experimental studies have been carried out on atom-sized contacts of metals and alloys to uncover their unique mechanical and electronic properties. ${ }^{1}$ One remarkable characteristic of atomsized contacts is their ability to hold high current densities. For example, a monatomic chain of $\mathrm{Au}$ atoms can sustain current density as high as $8 \times 10^{10} \mathrm{~A} / \mathrm{cm}^{2}{ }^{2}$ Apart from its practical importance in nanoscale electronics, such a high current-carrying capacity also poses an interesting problem of stability of atom-sized contacts under high-bias (highcurrent) conditions. In the past few years, some studies have been done on a variety of high-bias (high-current) phenomena in atom-sized contacts, such as bias- (current)-induced forces and bond weakening (breaking), ${ }^{3-9}$ heat dissipation, ${ }^{9-12}$ two-level fluctuations in conductance, ${ }^{13-15}$ current-induced local oxidation, ${ }^{16}$ current-induced atom migration, ${ }^{17}$ and electromigration. ${ }^{18}$ However, most of these studies deal with contacts consisting of a single atom or a monatomic chain, and little has been known about stability of slightly larger contacts with conductance ranging from a few to several tens of $G_{0}\left(G_{0} \equiv 2 e^{2} / h\right.$ is the quantum unit of conductance). In our previous experiments, ${ }^{19,20}$ we used a type of conductance fluctuation in breaking contacts of noble metals, which bursts out when the conductance becomes $\sim(10-50) G_{0}$. This contact instability has a threshold current density of the order of $10^{10} \mathrm{~A} / \mathrm{cm}^{2}$ and hence must be current induced. However, our previous experiments are limited to contacts of $G>10 G_{0}$, because conductance fluctuations become hardly observable in smaller contacts of $G<10 G_{0}$ at low biases. ${ }^{19}$

In this paper, we take a different approach and focus our attention on contact break. Although the break of atom-sized contacts is intimately related to their contact instability, it has been little studied experimentally in the past. Most recently, Smit et al. ${ }^{21}$ created monatomic chains of $\mathrm{Au}$ and $\mathrm{Pt}$ at $4 \mathrm{~K}$ and measured their break voltage by increasing the contact bias until the chain ruptures. Alternatively in this work, we reduced the contact size by mechanically stretching a macroscopic contact and detected a break conductance at which the contact breaks. We investigated the bias (current) dependence of the break conductance and revealed that the contact break of noble metals changes from the break due to the current-induced instability to the one through a couple of preferred contact geometries.

\section{EXPERIMENTS}

As in our previous experiments, ${ }^{19}$ we employed a wiredisk contact consisting of a $0.5 \mathrm{~mm}$ diameter mechanically cut metal wire and a $9.5 \mathrm{~mm}$ diameter metal disk, both of which are of $99.999 \%$ purity. A schematic diagram of our experimental setup is illustrated in Fig. 1. The wire is mounted onto a tubular piezoelectric transducer (PZT) scanner of a commercial scanning tunnel microscope (STM) approach module (Burleigh ARIS-10-0.5). By applying a trian-

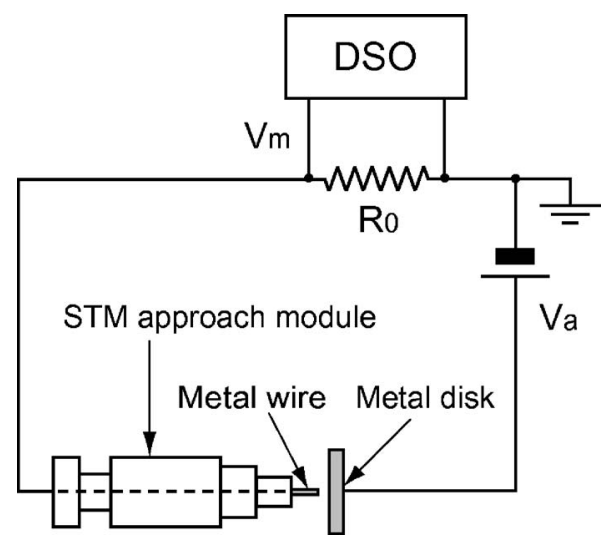

FIG. 1. Schematic of our experimental setup. A contact is made and broken repeatedly between a metal wire and a disk by oscillating the wire against the disk using a tubular PZT scanner. Transient conductance upon contact break is recorded by a fast digital storage oscilloscope (DSO), which monitors voltage drop across a currentsensing resistor $R_{0}$ connected in series with the contact. 


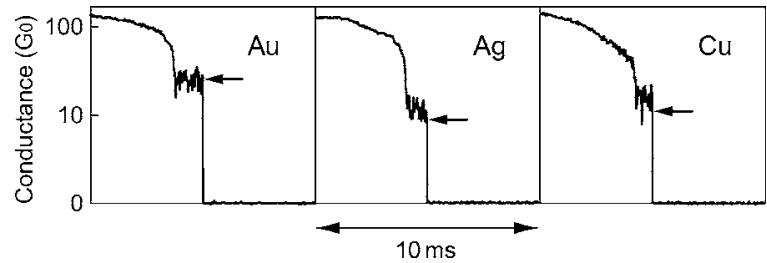

FIG. 2. Examples of transient conductance trace recorded on $\mathrm{Au}, \mathrm{Ag}$, and $\mathrm{Cu}$ contacts at $V_{a}=2.4 \mathrm{~V}$. The conductance scale is strongly nonlinear reflecting the nonlinear signal-to-conductance conversion in our measurements. In each trace, the conductance shows appreciable fluctuation before the contact breaks off. Arrows indicate locations of the break conductance $G_{b}$.

gular driving signal to the tube scanner, the wire continuously oscillates at $1 \mathrm{~Hz}$ along the normal direction of the disk surface. The estimated speed of the wire is $420 \mathrm{~nm} / \mathrm{s}$. While keeping the wire oscillating, we first crushed the wire against the disk and then adjusted the wire position so that the wire-disk contact repeats make (break) cycles at $1 \mathrm{~Hz}$. All measurements were carried out at room temperature in ultrahigh vacuum better than $5 \times 10^{-8} \mathrm{~Pa}$.

We measured a transient conductance at each contact break by monitoring a voltage drop $V_{m}$ across a currentsensing resistor $R_{0}=1 \mathrm{k} \Omega$ connected in series with the contact as shown in Fig. 1. A fast digital oscilloscope (Tektronix TDS3052) was used to record $V_{m}$ with a sampling time interval of $20 \mu \mathrm{s}$. We employed a rather coarse time resolution to observe the entire time evolution of the conductance. In our setup, a contact conductance $G$ is nonlinearly related to $V_{m}$ through a relation $G=V_{m} /\left(V_{a}-V_{m}\right) R_{0}$. Both contact bias and current, denoted by $V_{b}$ and $I$, also vary with the conductance as $V_{b}=V_{a} /\left(1+G R_{0}\right)$ and $I=G V_{a} /\left(1+G R_{0}\right)$, respectively. Note that when $G R_{0} \gg 1$, the contact current becomes $I \sim V_{a} / R_{0}$, so that changing $V_{a}$ is effectively equivalent to changing $I$.

Figure 2 shows typical conductance traces of noble metal contacts observed at $V_{a}=2.4 \mathrm{~V}$. A strongly nonlinear conductance scale in Fig. 2 is due to the nonlinear $G-V_{m}$ relation mentioned above. In each trace, the conductance first de- creases rather smoothly with time and suddenly shows a short fluctuation before the conductance jumps to zero. The conductance fluctuations seen in Fig. 2 are apparently the same fluctuation that we observed in our previous experiments. ${ }^{19,20}$ We defined and measured the break conductance $G_{b}$ as the conductance of the last data point before the conductance drops below $0.5 G_{0}$. Arrows in Fig. 2 indicate locations of $G_{b}$ of traces shown in the figure.

We varied $V_{a}$ and investigated how $G_{b}$ changes with $V_{a}$. Different voltage steps were employed depending on the magnitude of $V_{a}: 0.1 \mathrm{~V}$ step from $V_{a}=0.1 \mathrm{~V}$ through $1.0 \mathrm{~V}$, and $0.4 \mathrm{~V}$ step from $1.2 \mathrm{~V}$ through $3.2 \mathrm{~V}$. In one series of measurement, we recorded 200 traces at each $V_{a}$ and repeated the series 10 times to obtain 2000 traces in total. After finishing one series of measurements, we separated away the wire-disk contact and reestablished the contact to start the next series. This data-taking procedure may help average out effects of possible contact degradation during measurements. We carried out two experiments on different wire-disk samples (the same disk was used but repolished) and found no serious differences between experimental results.

\section{EXPERIMENTAL RESULTS AND DISCUSSION}

\section{A. Break conductance under high biases (currents)}

Figure 3 summarizes histograms of $G_{b}$ obtained on noble metal contacts for $V_{a} \geqslant 1.2 \mathrm{~V}$. Each histogram was constructed from 2000 conductance traces. Note that the bin width is not uniform among histograms and increases with $V_{a}$. This happens because we first made histograms of $V_{m}$ and converted them to $G_{b}$ histograms using the nonlinear $G-V_{m}$ relation described in Sec. II. The same relation also results in a lower $S / N$ ratio and larger background in the higher conductance side of each histogram. These effects are particularly noticeable in histograms for $V_{a}>2.4 \mathrm{~V}$.

We first note that the distribution of $G_{b}$ extends over $\sim(10-60) G_{0}$, showing that noble metal contacts typically break in this conductance range. Because the magnitude of $G_{b}$ is in the semiclassical regime, we can use the Sharvin formula ${ }^{22}$ for $G_{b}$ to estimate the contact size at the contact (a) Au contacts



(b) Ag contacts

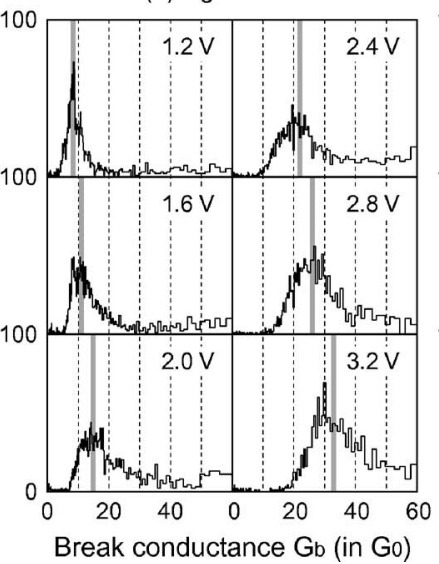

(c) Cu contacts

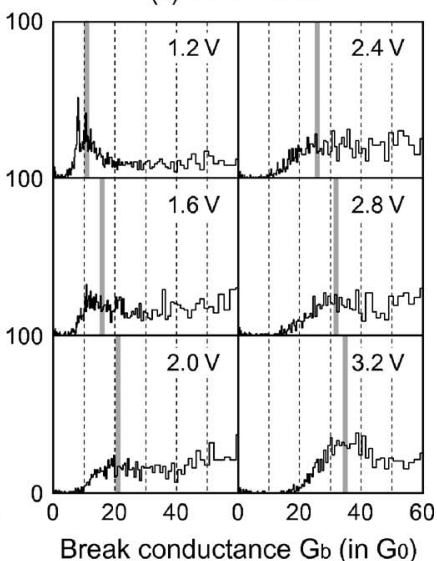

FIG. 3. Break conductance histograms of noble metal contacts obtained at $V_{a} \geqslant 1.2 \mathrm{~V}$. Each histogram shows a broad peak structure, the position of which is indicated by a gray line. 
break. For a circular constriction with radius $R$, the Sharvin conductance can be expressed as

$$
G=\frac{\left(k_{F} R\right)^{2}}{4} G_{0}=\frac{k_{F}^{2} A}{4 \pi} G_{0},
$$

where $k_{F}$ is the electron Fermi wave vector, and $A=\pi R^{2}$ is a cross-sectional contact area. By substituting $G_{b}$ $\sim(10-60) G_{0}$ into Eq. (1), we obtain $R \sim(0.5-1.3) \mathrm{nm}$. Thus, noble metal contacts break off after their size is reduced to a couple of $\mathrm{nm}$ or less. However, note that our estimation of $A$ and $R$ (and hence the contact current density $\hat{j}_{b}$ to be discussed below) entirely depends on Eq. (1), the validity of which has been little tested experimentally. ${ }^{23}$ Reliable contact size estimation can only be made after establishing a proper (conductance)-(contact size) relation, probably through direct transmission-electron-microscopy observations of nanocontacts.

All histograms in Fig. 3 display a broad single-peak structure. The peak is well visible in $\mathrm{Ag}$ histograms but somewhat obscured by the background in $\mathrm{Au}$ and $\mathrm{Cu}$ histograms at $V_{a}>2.0 \mathrm{~V}$. This single-peak distribution of $G_{b}$ clearly indicates that noble metal contacts break off not at arbitrary sizes but at a preferred contact size corresponding to the peak conductance. We hereafter denote this peak position by $\hat{G}_{b}$. To obtain $\hat{G}_{b}$ quantitatively, we carried out a Gaussian peak fitting for each histogram in Fig. 3. In histograms where the peak is broadened by the background, we assumed a power law $\alpha\left(G_{b}\right)^{\beta}$ for the background and determined parameters $\alpha$ and $\beta$ by least-squares fitting. In this background fitting, an extended histogram covering $G_{b}$ up to $100 G_{0}$ was used for displaying the background more clearly. After subtracting the background, the histogram exhibits a well-defined peak, the position of which can be determined by the Gaussian peak fitting. A gray thick line in each histogram indicates the position of the resulting $\hat{G}_{b}$.

It can be seen in Fig. 3 that all $\hat{G}_{b} \partial \mathrm{s}$ of three noble metals shift to the low conductance side with decreasing $V_{a}$. This is not, however, a bias effect because $\hat{G}_{b} R_{0}>1$ and it is not the bias but the contact current $I$ that varies with $V_{a}$, as we pointed out in Sec. II. Thus, the observed shift of $\hat{G}_{b}$ is actually a current-induced shift. We found that $\hat{G}_{b}$ decreases nearly proportionally to $I$, and the ratio $I / \hat{G}_{b}$ remains almost constant. Because $\hat{G}_{b}$ is proportional to a contact area $A_{b}$ according to Eq. (1), the constant $I / \hat{G}_{b}$ means a constant current density $I / A_{b}$. To check this point, we calculated $A_{b}$ by substituting $\hat{G}_{b}$ into Eq. (1) and estimated the current density at the contact break $\hat{j}_{b}=I / A_{b}$. Filled circles in Figs. 4(a)-4(c) at $I \gtrsim 1 \mathrm{~mA}$ represent the resulting $\hat{j}_{b}$ for $\mathrm{Au}, \mathrm{Ag}$, and $\mathrm{Cu}$, respectively. In this high current regime, all $\hat{j}_{b} \partial \mathrm{s}$ of three noble metals stay nearly constant. This constant $\hat{j}_{b}$ defines a critical current density $j_{c}$ for contact break. It should be noted here that this $j_{c}$ is a statistical property and that not all contacts necessarily break at $j_{c}$. Physically, it represents the current density at which a contact breaks with maximum likelihood. From the average $\hat{j}_{b}$ for $I \gtrsim 1 \mathrm{~mA}$, we obtain 6

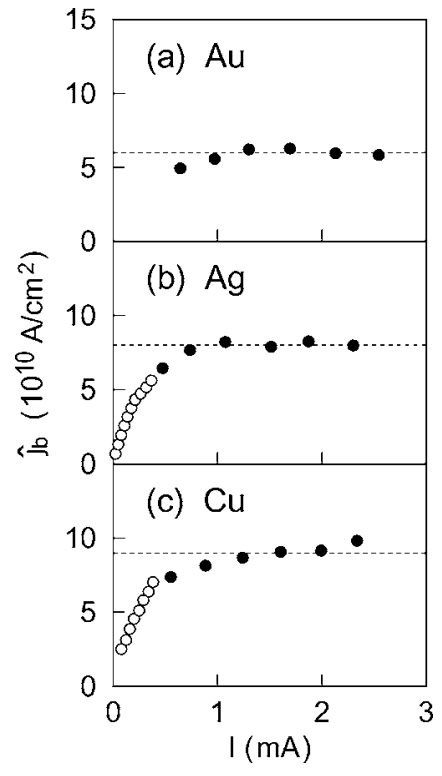

FIG. 4. Current density $\hat{j}_{b}$ at contact break estimated from $\hat{G}_{b}$ for (a) $\mathrm{Au}$, (b) $\mathrm{Ag}$, and (c) $\mathrm{Cu}$. Filled and empty circles represent $\hat{j}_{b} \partial \mathrm{s}$ corresponding to $\hat{G}_{b} \partial \mathrm{s}$ shown by gray lines in Figs. 3 and 5, respectively. A dashed line in each plot indicates a critical current density $j_{c}$ for the current-induced contact break.

$\times 10^{10}, 8 \times 10^{10}$, and $9 \times 10^{10} \mathrm{~A} / \mathrm{cm}^{2}$ for $j_{c}$ of $\mathrm{Au}, \mathrm{Ag}$, and $\mathrm{Cu}$, respectively. These $j_{c}$ values of noble metals are in excellent agreement with their threshold current densities for the conductance fluctuations obtained in our previous work. ${ }^{19,20}$ This coincidence is not unexpected because the conductance fluctuation usually precedes the contact break, as shown in Fig. 2. The current-induced contact instability that causes the conductance fluctuation is thus also responsible for the contact break of noble metals at $I \gtrsim 1 \mathrm{~mA}$.

Considering the high level of $j_{c}$, it is natural to assume high-current phenomena such as electromigration or local melting for a possible mechanism of the contact instability. ${ }^{19,20}$ Because the activation energy of electromigration in a bulk contact roughly scales with the melting temperature $T_{m}$ of a contact metal, ${ }^{24}$ either electromigration or local melting would predict positive correlation between $j_{c}$ and $T_{m}$. Although our result $j_{c, \mathrm{Au}}<j_{c, \mathrm{Ag}}<j_{c, \mathrm{Cu}}$ roughly conforms the speculated correlation, our preliminary experiments on $\mathrm{Al}$ and $\mathrm{Pt}$ contacts $^{25}$ suggest that the correlation holds for Al but not for Pt. Thus, the problem is still open, and further experiments on $j_{c}$ of other metals and direct microscopy observations of atom-sized contacts under high currents ${ }^{18}$ should be necessary for nailing down the underlying instability mechanism.

It is worth noting that the ratio $I / \hat{G}_{b}$ represents a typical break voltage of a contact and can be directly compared to experimental data obtained by Park et al. ${ }^{26}$ They passed high current through thin-film Au nanowires and measured the voltage at the wire failure. For contact currents $\sim(2.5-15) \mathrm{mA}$, they found that majority of nanowires break at a constant voltage $\sim 0.5 \mathrm{~V}$. This break voltage nicely coincides with our $I / \hat{G}_{b} \sim 0.65 \mathrm{~V}$ for Au. Because their lithographically defined thin-film nanowires differ in many re- 
(a) Au contacts

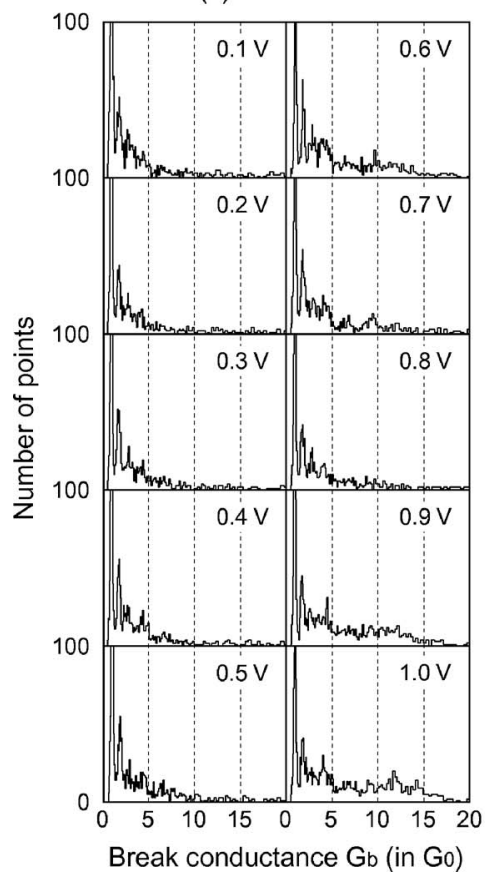

(b) Ag contacts

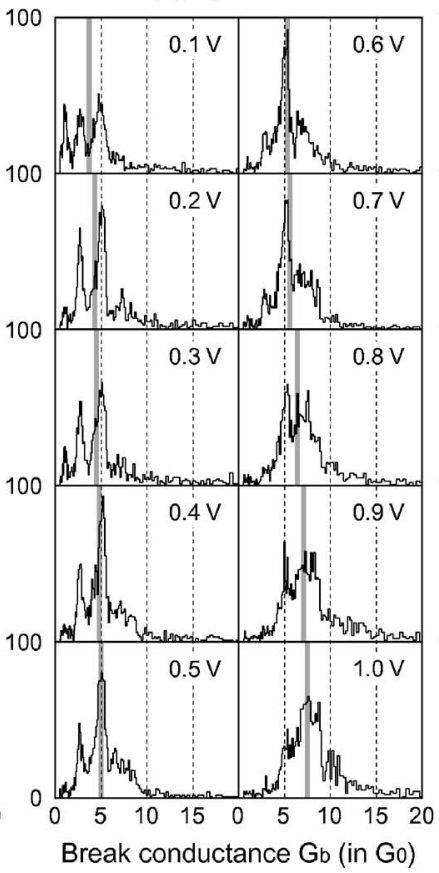

(c) Cu contacts

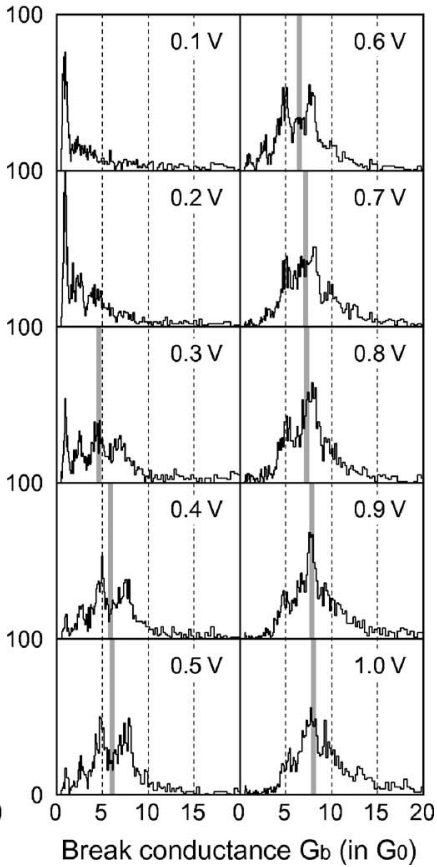

FIG. 5. Break conductance histograms of noble metal contacts obtained at $V_{a} \leqslant 1.0 \mathrm{~V}$. Histograms show a couple of sharp peaks corresponding to certain stable contact geometries. Gray strip lines indicate values of $\hat{G}_{b}$ of the current-induced break, which weakly influences the break of $\mathrm{Ag}$ and $\mathrm{Cu}$ contacts as explained in the text.

spects from our wire-disk breaking contacts, a possibility of fortuitous coincidence cannot be ruled out. Nevertheless, we believe that the current-induced break at constant $j_{c}$ is a robust characteristic of metals and that nanosized contacts of various sizes and geometries generally break at $j_{c}$ (with maximum likelihood). Similar break voltage measurements on $\mathrm{Ag}$ and $\mathrm{Cu}$ thin-film nanowires will clarify the issue.

\section{B. Break conductance under low biases (currents)}

When $V_{a}$ decreases below $1.0 \mathrm{~V}, \hat{G}_{b}$ histograms become more structured and exhibit a few sharp peaks as seen in Figs. 5(a)-5(c). In the case of $\mathrm{Au}$, a pronounced sharp peak [overscaled in all Au histograms in Fig. 5(a)] appears at $1 G_{0}$ with a couple of subpeaks at $2 G_{0}$ and $3 G_{0}$. After the appearance of this large $1 G_{0}$ peak, the histogram barely changes with $V_{a}$. Most Au contacts break off at the $1 G_{0}$ contact regardless of the contact bias (current). Because the $1 G_{0}$ peak already emerges in the $1.2 \mathrm{~V}$ histogram in Fig. 3(a), there is a transition at $V_{a} \sim(1.0-1.2) \mathrm{V}$ in the contact break mode of $\mathrm{Au}$ from the current-induced break at $j_{c}$ to the preferential break at $1 G_{0}$. According to Smit et al., ${ }^{21}$ a typical break voltage of $1 G_{0}$ single-atom chains of $\mathrm{Au}$ is $1.2 \mathrm{~V}$ at $4 \mathrm{~K}$. Though the break voltage at room temperature would be lower than that at $4 \mathrm{~K}$, it seems likely that the $1 G_{0}$ contact of $\mathrm{Au}$ at $V_{a}<1.0 \mathrm{~V}$ should have sufficient stability to stand against the current-induced contact break and make the $1 G_{0}$-contact break as a dominant break mode.

Sharp peaks of stable contact geometries can also be observed in $\mathrm{Ag}$ and $\mathrm{Cu}$ histograms in Figs. 5(b) and 5(c). For $V_{a}>0.2 \mathrm{~V}$, they appear at $7.5 G_{0}, 5 G_{0}$, and $2.5 G_{0}$. This pref- erential break of $\mathrm{Ag}$ and $\mathrm{Cu}$ contacts at $(2.5-7.5) G_{0}$ reduces their chance to deform into the $1 G_{0}$ contact and probably accounts for the observed small formation probability of the $1 G_{0}$ contact in $\mathrm{Ag}$ and $\mathrm{Cu}$ for $V_{a}>0.2 \mathrm{~V} \cdot{ }^{19,20,27}$ In Figs. 5(b) and $5(\mathrm{c})$, there can be seen a systematic height variation with $V_{a}$ among these sharp peaks. The $7.5 G_{0}$ peak of $\mathrm{Ag}$, for example, constitutes a main peak at $1.2 \mathrm{~V}$. Then, the $5 G_{0}$ peak starts to grow up as $V_{a}$ decreases and becomes a dominant peak for $0.4-0.8 \mathrm{~V}$. On the other hand, the $2.5 G_{0}$ peak emerges at $0.6 \mathrm{~V}$ and catches up with the $5 G_{0}$ peak at $0.2 \mathrm{~V}$. Finally, the $1 G_{0}$ peak appears at $0.4 \mathrm{~V}$. This suggests a tendency that a sharp peak of lower conductance acquires more intensity with decreasing $V_{a}$. Similar peak-height evolution can also be found on sharp peaks in $\mathrm{Cu}$ histograms for $V_{a}$ $>0.2 \mathrm{~V}$. By noting the resemblance between this shift of the peak intensity and that of $\hat{G}_{b}$, we consider that the broad peak structure of the current-induced break [seen in Figs. $3(\mathrm{~b})$ and 3(c)] continues to exist at low $V_{a}$ but becomes entirely "modulated" by sharp peaks of stable geometries. To determine its peak position $\hat{G}_{b}$ of the modulated broad peak, we again assumed a Gaussian profile and carried out a leastsquares fit of it to the envelope of sharp peaks. The resulting $\hat{G}_{b}$ is indicated by gray thick lines in some histograms of $\mathrm{Ag}$ and $\mathrm{Cu}$ in Figs. 5(b) and 5(c), respectively. ${ }^{28}$ From the comparison of Fig. 5 and Fig. 3, we can see that the shift of $\hat{G}_{b}$ at high $V_{a}$ continuously extends to the low $V_{a}$ regime, but significantly slows down. Because of this saturating behavior of $\hat{G}_{b}$, the effective critical current density $\hat{j}_{b}$ calculated from $\hat{G}_{b}$ deviates from the constant $j_{c}$ and decreases with $I$ as shown by empty circles in Figs. 4(b) and 4(c). Probably, the current-induced instability at low biases (currents) becomes 
too weak to keep the linear shift of $\hat{G}_{b}$. It only makes a secondary effect on the contact break through modifying the relative preference of stable contact geometries at the contact break.

When $V_{a}$ decreases down to $0.2 \mathrm{~V}$, the $\mathrm{Cu} 1 G_{0}$ peak suddenly grows up and dominates the histogram as seen in Fig. 5(c). Thus, the break mode of $\mathrm{Cu}$ contacts makes the second transition to the $1 G_{0}$ contact break at $V_{a} \sim 0.2 \mathrm{~V}$. The same transition happens in Au contacts at $V_{a} \sim 1.2 \mathrm{~V}$, but cannot be observed in $\mathrm{Ag}$ contacts at least down to $0.1 \mathrm{~V}$. These results imply the following inequality for the high-bias stability of $1 G_{0}$ contacts of noble metals that $\mathrm{Au}>\mathrm{Cu}>\mathrm{Ag}$. Further measurements on the break voltage of $\mathrm{Ag}$ and $\mathrm{Cu}$ $1 G_{0}$ contacts will clarify the validity of this stability relation.

Sharp peaks in Fig. 5 correspond to certain contact geometries that are preferred at the contact break and hence should be more stable than others. Because sharp peaks in a conventional conductance histogram also represent stable contact geometries, we can expect similar peak structures for our low $V_{a}$ break conductance histograms and conventional conductance histograms. In fact, the pronounced $1 G_{0}$ peak and small subpeaks in Fig. 5(a) reproduce well the features of a typical conductance histogram of Au. Also, $2.5 G_{0}$ and $5 G_{0}$ peaks in Figs. 5(b) and 5(c) might correspond to $2.4 G_{0}$ and (4-4.5) $G_{0}$ peaks, respectively, reported in conventional conductance histograms of $\mathrm{Ag}$ and $\mathrm{Cu} .{ }^{29,30}$ However, these conductance peaks are not observed in other literature, ${ }^{1}$ and the peak correspondence between break and conventional conductance histograms remains unclear for $\mathrm{Ag}$ and $\mathrm{Cu}$.

\section{CONCLUSION}

Our break conductance measurements have revealed two different break modes in noble metal contacts. Under high currents, a contact undergoes the current-induced break, where the contact breaks most likely when the current density reaches a critical value. The estimated critical current density ranges $(6-9) \times 10^{10} \mathrm{~A} / \mathrm{cm}^{2}$ for noble metals. These values are in excellent agreement with those of the threshold current density for the onset of the conductance fluctuation. Thus, the current-induced contact instability that generates the conductance fluctuation is also responsible for the contact break. At low biases (currents), the break mode changes to another mode where a contact tends to break at one of its stable contact geometries. In the case of $\mathrm{Au}$, a contact always ruptures at $1 G_{0}$, and this $1 G_{0}$ contact break is unaffected by the contact bias (current). On the other hand, most Ag and $\mathrm{Cu}$ contacts break at $7.5 G_{0}, 5 G_{0}$, and $2.5 G_{0}$ contacts, and their relative preference is weakly influenced by the currentinduced instability. Our experimental results suggest that the $1 G_{0}$ contact of Au has superior stability against the currentinduced instability than other contact geometries of $\mathrm{Ag}$ and $\mathrm{Cu}$ including their $1 G_{0}$ contacts.

\section{ACKNOWLEDGMENT}

This work was supported by the Grant-in-Aid for Scientific Research (B) No. 14340091 from Japan Society for the Promotion of Science.
*Corresponding author. Email address: sakai@iic.kyoto-u.ac.jp

${ }^{1}$ N. Agraït, A. Levy Yeyati, and J. M. van Ruitenbeek, Phys. Rep. 377, 81 (2003).

${ }^{2}$ A. I. Yanson, G. Rubio-Bollinger, H. E. van den Brom, N. Agraï, and J. M. van Ruitenbeek, Nature (London) 395, 783 (1998).

${ }^{3}$ T. N. Todorov, J. Hoekstra, and A. P. Sutton, Philos. Mag. B 80, 421 (2000).

${ }^{4}$ T. N. Todorov, J. Hoekstra, and A. P. Sutton, Phys. Rev. Lett. 86, 3606 (2001).

${ }^{5}$ M. Di Ventra and N. D. Lang, Phys. Rev. B 65, 045402 (2001).

${ }^{6}$ M. Di Ventra, S. T. Pantelides, and N. D. Lang, Phys. Rev. Lett. 88, 046801 (2002).

${ }^{7}$ M. Brandbyge, K. Stokbro, J. Taylor, J.-L. Mozos, and P. Ordejon, Phys. Rev. B 67, 193104 (2003).

${ }^{8}$ M. Di Ventra, Y. C. Chen, and T. N. Todorov, Phys. Rev. Lett. 92, 176803 (2004).

${ }^{9}$ Z. Yang, M. Chshiev, M. Zwolak, Y.-C. Chen, and M. Di Ventra, Phys. Rev. B 71, 041402(R) (2005).

${ }^{10}$ T. N. Todorov, Philos. Mag. B 77, 965 (1998).

${ }^{11}$ M. J. Montgomery, T. N. Todorov, and A. P. Sutton, J. Phys.: Condens. Matter 14, 5377 (2002).

${ }^{12}$ Y. C. Chen, M. Zwolak, and M. Di Ventra, Nano Lett. 3, 1691 (2004)

${ }^{13}$ H. E. van den Brom, A. I. Yanson, and J. M. van Ruitenbeek, Physica B 252, 69 (1998).

${ }^{14}$ A. Halbritter, Sz. Csonka, O. Yu. Kolesnychenko, G. Mihály, O.
I. Shklyarevskii, and H. van Kempen, Phys. Rev. B 65, 045413 (2002).

${ }^{15}$ Sz. Csonka, A. Halbritter, G. Mihály, E. Jurdik, O. I. Shklyarevskii, S. Speller, and H. van Kempen, Phys. Rev. Lett. 90, 116803 (2003).

${ }^{16}$ T. Schmidt, R. Martel, R. L. Sandstrom, and Ph. Avouris, Appl. Phys. Lett. 73, 2173 (1998).

${ }^{17}$ J. W. T. Heemskerk, Y. Noat, D. J. Bakker, J. M. van Ruitenbeek, B. J. Thijsse, and P. Klaver, Phys. Rev. B 67, 115416 (2003).

${ }^{18}$ S. Fujisawa, T. Kikkawa, and T. Kizuka, Jpn. J. Appl. Phys., Part 2 42, L1433 (2003).

${ }^{19}$ A. Fujii, J. Mizobata, S. Kurokawa, and A. Sakai, e-J. Surf. Sci. Nanotech. 2, 125 (2004).

${ }^{20}$ A. Fujii, S. Kurokawa, and A. Sakai, e-J. Surf. Sci. Nanotech. 2, 155 (2004).

${ }^{21}$ R. H. M. Smit, C. Untiedt, and J. M. van Ruitenbeek, Nanotechnology 15, S472 (2004).

${ }^{22}$ A. Garcïa-Martïn, J. A. Torres, and J. J. Sáenz, Phys. Rev. B 54, 13448 (1996).

${ }^{23}$ D. Erts, H. Olin, L. Ryen, E. Olsson, and A. Tholen: Phys. Rev. B 61, 12725 (2000).

${ }^{24}$ A. Mogro-Campero, J. Appl. Phys. 53, 1224 (1982).

${ }^{25}$ T. Minowa, S. Kurokawa, and A. Sakai, Physica E (to be published); T. Minowa, M. Tsutsui, S. Kurokawa, and A. Sakai, Jpn. J. Appl. Phys. (to be published).

${ }^{26}$ H. Park, A. K. L. Lim, A. P. Alivisatos, J. Park, and P. L. 
McEuen, Appl. Phys. Lett. 75, 301 (1999).

${ }^{27}$ A. Fujii, A. Kusuda, J. Mizobata, S. Kurokawa, and A. Sakai, Thin Solid Films, 464-465, 251 (2004).

${ }^{28}$ To check the reliability of estimated $\hat{G}_{b}$ values, we also tried a nonlinear least-squares fitting to the distribution of $G_{b}$ assuming that it consists of a product of one broad Gaussian peak and a couple of narrow Gaussian peaks of equal height and width, representing the broad peak and sharp peaks, respectively. The resulting $\hat{G}_{b}$ values agree with those obtained by the simple envelope fitting within $10 \%$ and $20 \%$ for $\mathrm{Ag}$ and $\mathrm{Cu}$ contacts, respectively.

${ }^{29}$ V. Rodrigues, J. Bettini, A. R. Rocha, L. G. C. Rego, and D. Ugarte, Phys. Rev. B 65, 153402 (2002).

${ }^{30}$ J. C. González, V. Rodrigues, J. Bettini, L. G. C. Rego, A. R. Rocha, P. Z. Coura, S. O. Dantas, F. Sato, D. S. Galvão, and D. Ugarte, Phys. Rev. Lett. 93, 126103 (2004). 